\title{
Stereolithography and the Use of Pre-Adapted / Fabricated Plates for Accurate Repair of Maxillofacial Defects
}

Jehad Al-Sukhun ${ }^{* *}$ and Heikki Penttila ${ }^{2}$

${ }^{1}$ Department of Oral and Maxillofacial Surgery, Euro Oral LTD, Fredrikinkatu 41A 9, 00120 HUS, Helsinki, Finland

${ }^{2}$ Department of Physics, University of Jyvaskyla, Finland

\begin{abstract}
The purpose of this paper was to share our clinical experience on the use of pre-adapted / fabricated plates using stereolithography for accurate repair of maxillofacial defects. We present five cases where pre-adapted / fabricated plates were either used to reconstruct defects following tumour resection or for secondary reconstructions of maxillofacial trauma. Our opinion concerning Stereolithography Models (SMs) is that they probably do not offer the surgeon relevant diagnostics information, but they are very useful in the surgical planning of any maxillofacial operation. SMs allow direct simulation of osteotomies, grafts and the measurement of bone movements. Prefabricated/adapted plates combined with SMs reduce intra-operative time and morbidity, increase accuracy of the surgical reconstruction and minimize complications and surgeons' stress. However, these technologies are expensive. Therefore, considering their cost, we see indications in secondary corrections of the maxillofacial trauma and tumour reconstruction.
\end{abstract}

Keywords: Stereolithography; Maxillofacial trauma; Tumour reconstruction

\section{Introduction}

Functional reconstruction of the maxillofacial skeleton implies replication of the normal volume and contour of both soft and hard tissue to produce normal form and function of the face, mouth and jaws. Maxillofacial defects may result following ablative surgery for malignancy, trauma and corrective surgery for congenital facial deformities. Hence, when planning the reconstruction, the extent of the resection, type of the missing tissues, and the precise function and contour are all important considerations. Successful rehabilitation, following ablative surgery for malignancy is likely to depend on the preoperative fitness score as outlined by the American Joint Cancer Committee (AJCC), [1] and the associated surgical trauma of the resection and reconstruction. Stereolithography, a preoperative technique, accurately measures and contours a reconstruction plate for the placement at the time of the skeletal resection [2-8]. It significantly decreases operating time and increases the surgeon's option to manage complex reconstructions (Table 1). However, these technologies are expensive. Therefore, their use must be critically evaluated with regard to accuracy, informative value, cost and benefits in terms of improved diagnosis and better surgery. Stereolithography as a technique has been mainly used to fabricate a template for custom cranioplasty to cover defects in the skull [2-10]. Its application to the field of maxillofacial skeleton to fabricate and/or pre-adapt reconstructive plates has been limited to a few cases reported in the literature $[11,12]$. The purpose of this paper was to share our clinical experience on the use of preadapted/fabricated plates, using stereolithography, for accurate repair

1. Useful in the surgical planning of virtually any maxillofacial operation

2. Direct simulation of osteotomies, grafts and the measurement of bone movements

3. Reduce intra-operative time and morbidity

4. Increase accuracy of the surgical reconstruction and minimize complications

5. Reduce Surgeons' stress

6. Improved preoperative selection of implants and transplants

7. High informative value of SMs for teaching and patient information

8. Documentation of unusual cases.

Table 1: Advantages of Stereolithography models (SMs) in surgery. of maxillofacial defects. In this paper we present five cases where pre-adapted/fabricated plates were either used to reconstruct defects following tumour resection or for secondary reconstructions of maxillofacial trauma.

\section{Case One}

A 44-year-old man was referred to the division of the oral and maxillofacial surgery at the Helsinki University Hospital for the management of numbness of the lower lip and persistent dull pain in the lower left quadrant of the mandible, following a series of root canal fillings carried out on 35,36 and 37 . The medical history was unremarkable. Intra-oral examination did not reveal any evidence of swelling and the mucosa was non-tender, healthy and smooth. Extra orally, there was no swelling and the skin showed a normal texture with no fixation to the underlying tissues (Figure 1). CTscan and MRI imaging showed a homogeneous lesion occupying the left maxilla / left maxillary sinus and extending to affect the left part of the mandible inferiorly and the zygoma, orbit and anterior base of the skull superiorly. The histological features were consistent with a cribriform variant of adenoid cystic carcinoma. The size of the tumour and its anatomical complexity made it difficult to reconstruct the defect while maintaining optimal aesthetic and functional results. Therefore, pre-surgical planning was an important part of the operation. A model of the skull including the mandible was constructed using stereolithography (Figure 1). The model was then used to pre-adapt the reconstructive surgical plates to accurately bridge and restore the defects. There were minor anatomical differences between the model and the resected area. Therefore, the plates were slightly bent

*Corresponding author: Jehad Al-Sukhun, Department of Oral and Maxillofacial Surgery, Euro Oral LTD, Fredrikinkatu 41A 9, 00120 HUS, Helsinki, Finland, Tel: +358503071886; Fax: +3589483720; E-mail: jalsukhun@hotmail.com

Received July 22, 2013; Accepted August 25, 2013; Published August 27, 2013

Citation: Al-Sukhun J, Penttila H (2013) Stereolithography and the Use of Pre-Adapted / Fabricated Plates for Accurate Repair of Maxillofacial Defects. Anaplastology S6: 004. doi: 10.4172/2161-1173.S6-004

Copyright: (c) 2013 Al-Sukhun J, et al. This is an open-access article distributed under the terms of the Creative Commons Attribution License, which permits unrestricted use, distribution, and reproduction in any medium, provided the original author and source are credited. 
Citation: Al-Sukhun J, Penttila H (2013) Stereolithography and the Use of Pre-Adapted / Fabricated Plates for Accurate Repair of Maxillofacial Defects. Anaplastology S6: 004. doi: 10.4172/2161-1173.S6-004

and adapted to fit accurately to the remaining bone. The tumour was surgically resected along with a bilateral supra omohyoid neck dissection. The defect, after the hemifacectmoy, was reconstructed by combining two classical free flaps: 1) A Deep Circumflex Iliac Artery free (DCIA) flap and 2) A Free Fibular (FF) flap. The DCIA and FF grafts were fixed using the pre-adapted surgical plates (Figure 1). No references were found in the literature about the use of this technique for treating defects similar to those described in this paper. The patient was followed up at monthly intervals during the first six months and then progressively less frequently. A postoperative CT-scan and MRI were performed preoperatively, immediately after the surgery and one month postoperatively. Analysis of the aesthetic and functional results of this type of reconstruction was difficult since there were no objective parameters allowing us to evaluate them by standard criteria. Therefore, analysis was based on an interview with the patient, his family and other staff members of our department. Difficulties in performing any of the functions e.g. eating and speaking, were unremarkable. In terms of facial aesthetics, the results were very satisfactory. The colour and texture of the skin used enabled an adequate degree of facial harmony to be maintained. The final appearance of the scars was good. This was due to the technique that took advantage of facial cutaneous folds, and attempts to restore the relaxed skin tension lines. The second step would have been to fit ocular and dental prostheses. However, given the immediate need for complementary radiotherapy, it was decided to defer this treatment until later.

\section{Case Two}

A 60 -year-old man with a 5-cm mass in the upper right hard palate was initially seen at the department of oral and maxillofacial surgery at Helsinki University Central Hospital. A CT-scan confirmed a large mass in the right antrum with loss of orbital floor and invasion of the tumour to the middle base of the skull. Histology results confirmed a mixed form of an adenoid cystic carcinoma. Through a WeberFerguson approach a right hemi-maxillectomy in continuation with craniotomy was carried out. A vertical ramus osteotomy in combination with a parasymphysial mandibular osteotomy was also performed to enhance access to the base of the skull with minimal

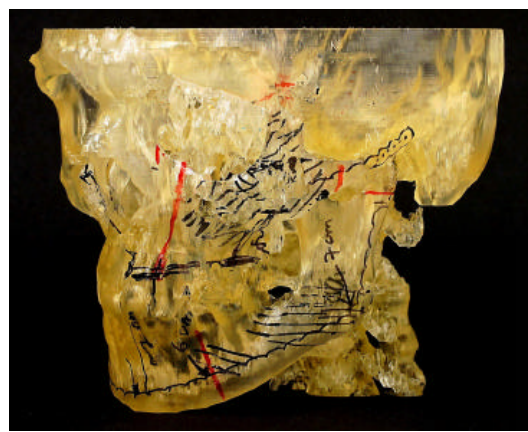

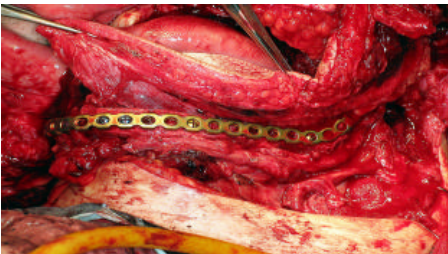

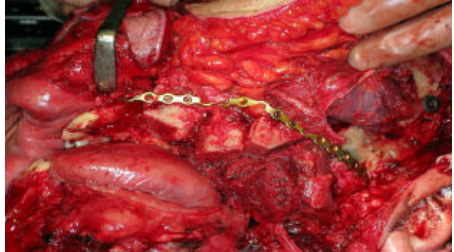

C

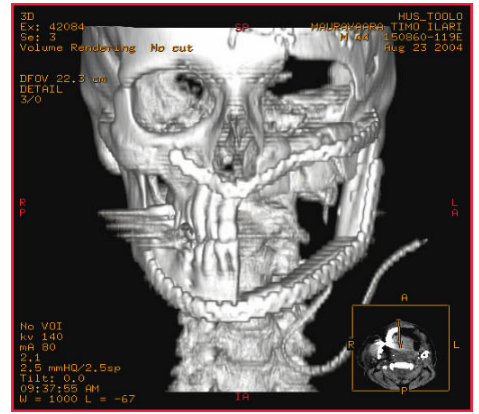

Figure 1: (A) Lateral view of the resin model fabricated using stereolithography. Red marks identified cuts for the osteotomies. Black lines indicated the extent of the tumour and the proposed location of the pre-adapted reconstructive plates. (B) The free fibular graft was fixed using a pre-contoured surgical plate to reconstruct the mandible. (C) The DCIA graft was fixed using a pre-contoured surgical plate to reconstruct the orbito-cutaneous and mid facial defects. (D) Postoperative, three dimensional formatted image displaying the defect after the resection of the tumour and the pre-adapted reconstructive surgical plates in place. The plates produced the exact contour of the maxilla and the mandible and maintained skeletal symmetry. 
morbidity. A left supra-omohyoid neck dissection with preservation of the neck vessels was included in this ablation. A DCIA flap with internal oblique was harvested from the ipsi-lateral side. The bone was vertically oriented and plated into position to replace the maxillary buttress, the anterior maxillary wall and antrum. The orbital rime was reconstructed using a free calvarial bone. The muscle was passed medially into the palatal defect and sutured to the lower eyelid skin superior to the graft. A reconstructive plate was pre-adapted prior to surgery using a stereolithrographic model of the skull (Figure 2) and used to hold the DCIA graft in place to maintain the three-dimensional contour of the mid-facial skeleton. A titanium mesh was also precontoured and adapted to match the anatomy of the orbital floor and used to reconstruct the inferior orbital wall (Figure 2). A further plate was pre-adapted, prior to the surgery, to restore the mandible into its original contour following the neck dissection and the anastamosis. The facial artery was anastmosed end-to-end into the DCIA and the vein end-to-end into the facial vein. Facial swelling decreased in two weeks, postoperatively, and the patient made excellent progress after the surgery and was able to return home within five weeks.

\section{Case Three}

A 18-year old female was initially seen with an osteosarcoma lesion occupying most of the left maxilla (Figure 3). Using a Weber-Ferguson approach a subtotal maxillectomy was carried out without a formal neck dissection. A DCIA flap with internal oblique was harvested from the right hip. To determine the amount of bone needed to restore the defect, a cure acrylic model of the defect was pre-fabricated using a stereolithrographic model of the skull (Figure 3). Using the model, the bone was contoured to reproduce the pyriforms and the exact contour of the maxilla. Pre-adapted plates were used to hold the bone in place, in order, to produce the exact contour of the maxilla. No attempts were made to reconstruct the nasal floor, which was allowed to epithelialize. The facial artery was anastmosed end-to-end into the DCIA and the vein end-to-end into the facial vein. There were no immediate postoperative problems and the patient made excellent progress and was able to go home within five weeks.

\section{Case Four}

A 36-year-old female patient who suffered a unilateral, secondary, left orbital blow-out fracture, with a $3 \mathrm{~cm}^{2}$ defect in the lateral aspect of the orbital floor, was selected for this study. Standard clinical examination consisted of clinical controls preoperatively and at 1, 2, 4, 8, 16 and 36 weeks postoperatively. CT-scan imaging was performed preoperatively and 30 weeks postoperatively using a CT scanner (Siemens Somatom, Erlangen, Germany. A resin model of the skull including the right and left orbits was constructed using stereolithography. The model was then used to develop a custom-fabricated titanium plate of the left orbital floor using the standard prosthetic, alginate impression and fabrication techniques (Figure 1). The orbital cavity was explored through a lower eyelid approach. The plate was then placed subperiosteally and held in place using three ready made titanium screws (Figure 4). There were no complications postoperatively e.g. no signs of diplopia, enophthalmos, infection. The patient was pleased with the aesthetic outcome. This custom pre-fabricated surgical plate proved to be a simple and reliable method to repair large secondary defects of the orbital floor.

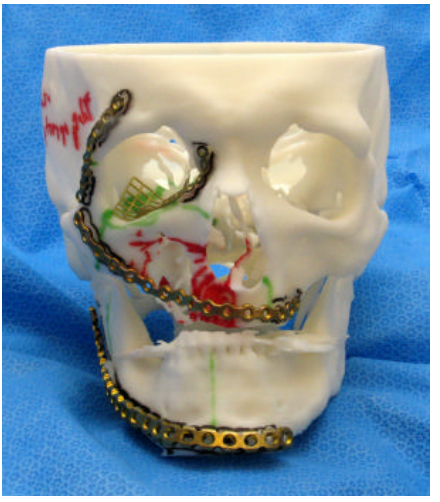

$\mathbf{A}$

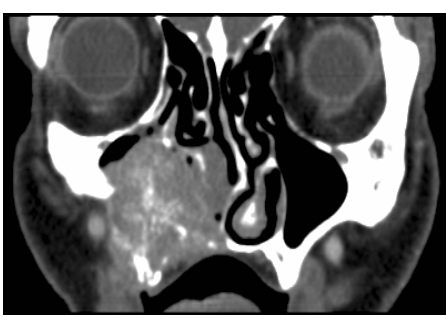

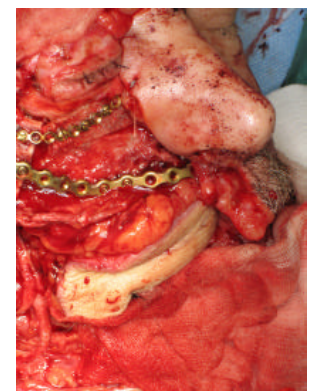

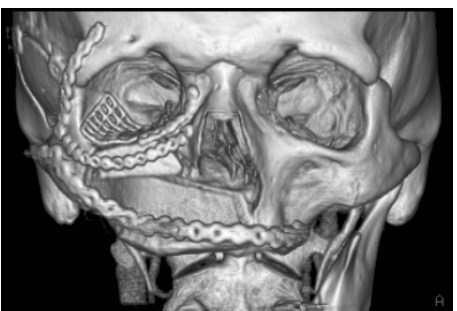

Figure 2: (A) Frontal view of the stereolithrography model with the pre-adapted plates in place. A titanium mesh was also pre-contoured and adapted to match the anatomy of the orbital floor and used to reconstruct the inferior orbital wall. (B) Frontal view showing the defect, reconstructed using free micro vascular grafts, with the pre-adapted reconstructive plates in place $(C)$ Preoperative MRI showing a homogenous mass occupying the left maxilla and sinus and extends to affect the orbital floor and base of the skull. (D) Postoperative, three dimensional formatted image displaying the defect after the resection of the tumour. The pre-adapted reconstructive surgical plates together with the titanium mesh bridged the defect, produced the exact contour of the maxilla and maintained skeletal symmetry. 
Citation: Al-Sukhun J, Penttila H (2013) Stereolithography and the Use of Pre-Adapted / Fabricated Plates for Accurate Repair of Maxillofacial Defects. Anaplastology S6: 004. doi: 10.4172/2161-1173.S6-004

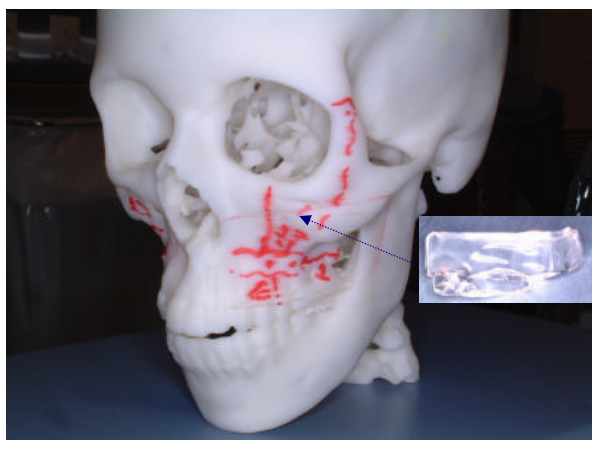

A

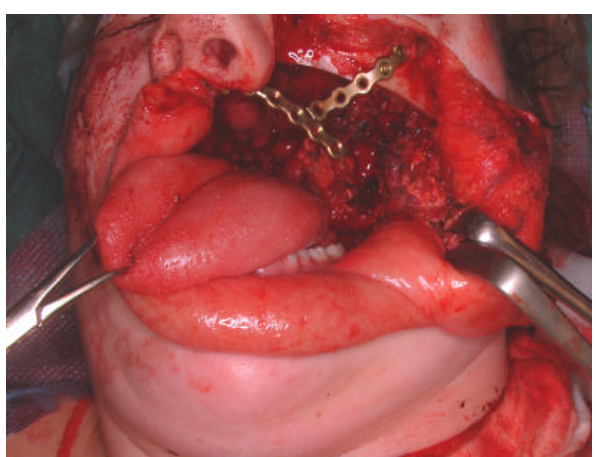

B

Figure 3: (A) Lateral view of the resin model fabricated using stereolithography. Red marks identified cuts for the osteotomies, the extent of the tumour and the proposed location of the pre-adapted reconstructive plates. Acrylic model of the defect was pre-fabricated and used to shape the DCIA graft to restore the contour of the maxilla and maintain facial symmetry. (B) Frontal view showing the defect after the resection with the pre-adapted reconstructive plates in place.

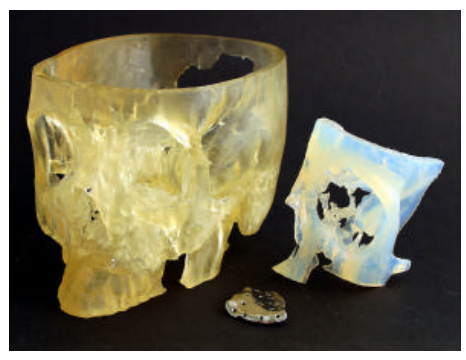

A

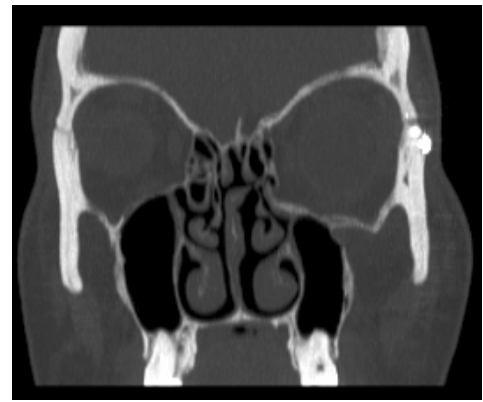

C

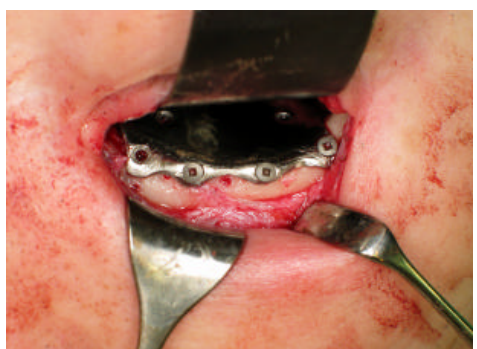

B

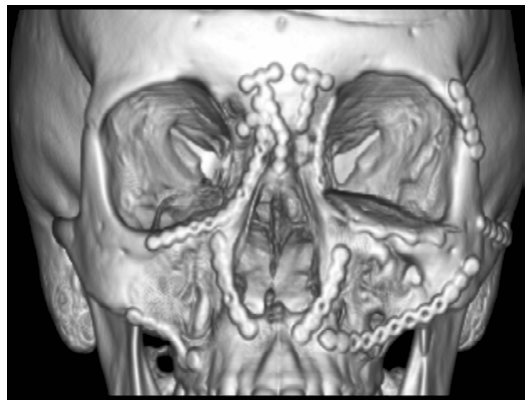

D

Figure 4: (A) Antero-lateral view of the stereolithography model with the pre-fabricated surgical plate. (B) The pre-fabricated plate was, accurately, fitted over the orbital defect and held in place using three ready made titanium screws. (C) A preoperative computed tomography scan (CT-scan) showing secondary left orbital blow-out fracture and enlarged orbital volume. (D) A postoperative three dimensional formatted image showing the position and shape of the plate on the orbital floor.

\section{Case Five}

A 33-year-old male patient who suffered unilateral, secondary, multiple left zygomatico-orbital fractures with a $2 \mathrm{~cm}^{2}$ defect in the lateral aspect of the orbital wall. The zygomatic-orbital complex was displaced inferiorly and posteriorly. A unicoronal flap was reflected to gain access to the lateral orbital wall and the zygomatic arch. Calvarial bone was harvested and used to restore the lateral orbital defect. The zygomatico-orbital complex was osteotomised as shown in Figure 5 and bone was repositioned in place using pre-adapted surgical plates for accurate reconstruction of the skeletal contour and restoring the facial symmetry (Figure 5). There were no complications postoperatively e.g. no signs of infection, facial assymetry etc. The patient was pleased with the aesthetic outcome. The ability to complete the non-surgical aspect of the patient's treatment in the laboratory allowed for precision not often achievable in an operative procedure.

\section{Summary and Discussion}

Stereolithography is a process that uses data generated from Computer-Assisted Design (CAD) to generate three-dimensional models. The data drives a laser over a bath of photosensitive resin 

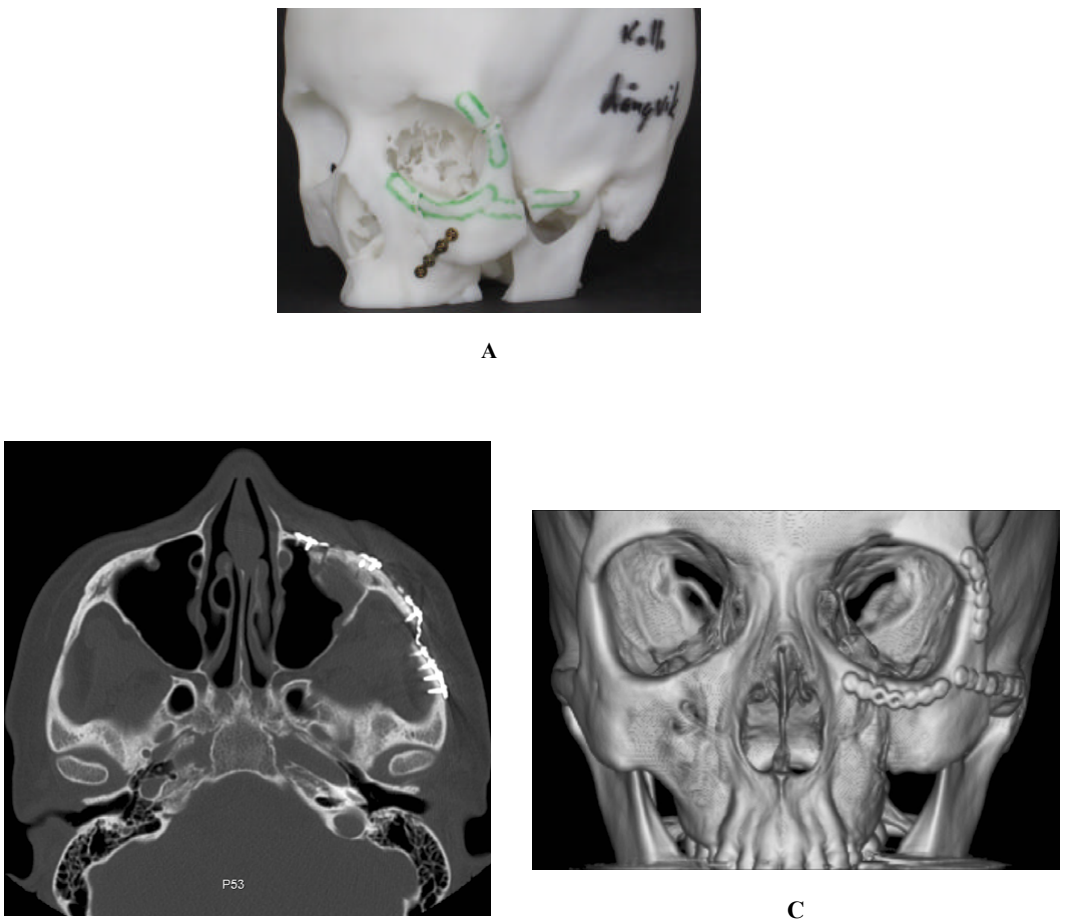

B

Figure 5: (A) Antero-lateral view of the resin model fabricated using stereolithography. Green marks identified the proposed location of the pre-adapted reconstructive plates. The model also demonstrates the cuts used to mobilize the zygomatico-orbital complex. The plate shown was only used for the purpose of holding the resected segment in place and not used in the final operation. (B) Preoperative CT-scan displaying the secondary depressed zygomatico-orbital complex. (C) Postoperative, three dimensional formatted image showing the pre-adapted reconstructive surgical plates in place. Their location exactly replicates the landmarks as out lined on the model preoperatively.

which produces a series of stacked slices, which produce an accurate three-dimensional resin model [5]. This technique can be used by the maxillofacial surgeon to produce three-dimensional representations of the facial bony structures using data from CT or MRI scans [210]. In particular, it allows the maxillofacial surgeon to appreciate spatial displacements in all three dimensions and to make accurate measurement of the deformity. However, prospective clinical studies are needed to demonstrate the value of stereolithographic modelling in reconstructive surgery [13]. The literature on stereolithography and its application in reconstructive surgery is limited to case reports and discussions about the feasibility of the technique [2-13]. There are no prospective studies demonstrating that the use of SMs improves the outcomes of reconstructive surgical procedures. Because of the lack of prospective clinical studies in the literature proving the value of SMs in reconstructive surgery, stereolithographic modelling is considered experimental and long term studies are welcomed to affirm the known usefulness of this relatively new technology in the field of reconstructive surgery.

The correction of post-traumatic facial asymmetry and defects following major ablation surgery to remove malignancies have always been difficult $[2,11]$. Great accuracy is required to achieve successful surgical results, due to the fact that facial deformity and asymmetry is often the result of relatively small magnitudes of bony displacement or deformity.

We were able to practise the surgery on the model, thus allowing full appreciation of the osteotomy bone cuts required to achieve the desired results. The ability to complete the non-surgical aspect of the patient's treatment in the laboratory, to pre-adapt or pre-fabricate surgical plates, ensured that the surgical accuracy achieved brought about a satisfactory outcome to the patient both functionally and aesthetically. SMs also provided an estimation of the volume of both bony and soft tissue structures for possible implantation and of bony cavities for reconstructive purposes (Case 3). Valuable theatre time was saved by allowing the pre-adapted / fabricated bone plates to be used for fixation on the post-operative SMs that demonstrate the planned realignment of the facial bones. Stereolethrography and pre-adapted / fabricated plates made these goals a reality.

The quality and accuracy of anatomical reproduction of the SMs, the presence of artefacts, as well as the informative value for diagnosis and surgical planning have been assessed on the basis of the subjective surgeons' experience. On the basis of our clinical practice, SMs resulted in accurate anatomic reproductions at least in the limits required for surgical applications. SMs may present over or under-contours in the reproduction of maxillofacial bones [10]. In general, the dimensional errors were within acceptable values. However, the defect that was commonly observed in these models was a poor reproduction of the dental elements, which was of no relevance to the reconstructive procedure.

Considering the informativevalue of these models, stereolithography has offered a clear diagnostic improvement, especially in complex post-traumatic, secondary corrections, like those involving the orbital region (Cases 4 and 5). However, the authors feel that it is important 
Citation: Al-Sukhun J, Penttila H (2013) Stereolithography and the Use of Pre-Adapted / Fabricated Plates for Accurate Repair of Maxillofacial Defects. Anaplastology S6: 004. doi: 10.4172/2161-1173.S6-004

Page 6 of 6

that surgeons are aware of the limited advantage of using SMs in the study of acute trauma cases. The increased cost and time required for these exams is not justified. Similarly, no substantial diagnostic benefit is obtained when applying the technique to benign jaw pathologies or tumours. Our opinion of SMs is that they probably do not offer the clinician relevant diagnostic information but they are very useful in the surgical planning of virtually any maxillofacial operation. SMs allow direct simulation of osteotomies, grafts and the measurement of bone movements. Pre-fabricated / adapted plates combined with SMs reduce the intra-operative time and morbidity, increase the accuracy of surgical reconstruction and minimize complications and surgeons' stress. Therefore, considering their cost, we see indications in secondary corrections of craniofacial trauma, tumour reconstruction, malformations and also in some preprosthetic-implant procedures. Finally, our clinical practice suggests a high informative value of SMs for teaching, patient information and documentation of unusual cases.

\section{Acknowledgement}

We would like to thank Mr Jarmo Nummenpää, medical illustrator, and Dr Anni Suomalainen, consultant radiologist, at the Helsinki University Central Hospital, for their technical expertise in providing the images and the photographs.

\section{References}

1. Beahrs OH, Henson DE, Hutter RVP, Myers MH (1988) Manual for Staging of Cancer. (3rdedn), Philadelphia, USA.

2. Joffe JM, McDermott PJ, Linney AD, Mosse CA, Harris M (1992) Computergenerated titanium cranioplasty: report of a new technique for repairing skull defects. Br J Neurosurg 6: 343-350.

3. Altobelli DE, Kikinis R, Mulliken JB, Cline H, Lorensen W, et al. (1993) Computer-assisted three-dimensional planning in craniofacial surgery. Plast Reconstr Surg 92: 576-585
4. Klimek L, Klein HM, Schneider W, Mösges R, Schmelzer B, et al. (1993) Stereolithographicmodelling for reconstructive head surgery. Acta Otorhinolaryngol Belg 47: 329-334.

5. Anderl H, ZurNedden D, Mühlbauer W, Twerdy K,Zanon E, et al. (1994) CTguided stereolithography as a new tool in craniofacial surgery. Br J Plast Surg 47: 60-64.

6. Rarnieri G, Bianchi SG, Spada MC (1999) Indications for the use of solid models for planning of craniomaxillofacial surgery. Phidas 2: 4-6.

7. Winder J, Cooke RS, Gray J, Fannin T, Fegan T (1999) Medical rapid prototyping and $3 \mathrm{D} \mathrm{CT}$ in the manufacture of custom made cranial titanium plates. J Med Eng Technol 23: 26-28.

8. D'Urso PS, Earwaker WJ, Barker TM, Redmond MJ, Thompson RG, et al. (2000) Custom cranioplasty using stereolithography and acrylic. Br J Plast Surg 53: $200-204$

9. Klein HM, Schneider W, Alzen G, Voy ED, Günther RW (1992) Pediatric craniofacial surgery: comparison of milling and stereolithography for 3D mode manufacturing. Pediatr Radiol 22: 458-460.

10. Perez-Arjona E, Dujovny M, Park H, Kulyanov D, Galaniuk A, et al. (2003) Stereolithography: neurosurgical and medical implications. Neurol Res 25 227-236.

11. Peckitt NS (1998) Stereolithography and the manufacture of customized implants in facial reconstruction: a flapless surgical technique. $\mathrm{Br} \mathrm{J}$ Oral Maxillofac Surg 36: 481.

12. Kernan BT, Wimsatt JA 3rd (2000) Use of a stereolithography model for accurate, preoperative adaptation of a reconstruction plate. J Oral Maxillofac Surg 58: 349-351.

13. Perlyn CA, Marsh JL, Vannier MW, Kane AA, Koppel P, et al. (2001) The craniofacial anomalies archive at St. Louis Children's Hospital: 20 years of craniofacial imaging experience. Plast Reconstr Surg 108: 1862-1870. 